\title{
Software Application for E-Health Monitoring System
}

\author{
Mr. Prasanna Puttaswamy ${ }^{1}$, Mr. Pramod Srivatsa Krishna Murthy ${ }^{2}$ \\ ${ }^{1,2}$ (M.Sc graduate from Coventry University, UK, Mysore, Karnataka)
}

\begin{abstract}
: this paper presents an application of system which monitors the condition of patients or an aged person continuously using WSN technology. E-Health monitoring system is integrated with medical sensor and small computing devices to communicate between medical centre and Patient. This system automatically alerts the medical centre in case of Emergency or if patient needs immediate Doctor Attention. Such an approach reduces the routine rounds of medical personal and allows them to concentrate on other important duties.
\end{abstract}

Keywords: Medical Sensors, E-Health Monitoring, Algorithm for E-Health monitoring, EHMS.

\section{Introduction}

Today's rapid growth in the technology has revolutionised many fields in the world, Medical field is being one of them. E-Health monitoring System's are effectively used in Monitoring of health condition of patient/ aged person remotely. This is achievable in this era due to the technological advancements in Wireless sensor Network's (WSN) which are widely used in E-Health monitoring System that are integrated with medical sensor to fetch the quest.

Wireless Sensor Network commonly referred as WSN is an emerging technology at present time. In a WSN, dozens or even hundreds of small computing devices are scattered in an environment [1]. Each computing devices are capable of performing different set of task/ function i.e. monitoring, displaying and sensing \& thus having broad commercial applications in Industries, Healthcare monitoring and military application and so on.

WSN device consist of a sensor or an actuator, a radio stack, embedded processor, an antenna and a battery. These devices are held within an enclosure [1].Generally all the WSNs use the sensor to sense or gather the data, then process those data for the subsequent use. In case of healthcare monitoring system the data will be processed in the sensor node if it is outside the safe zone, then the result will be transmitted to clinician or the concerned medical staff to respond to the patient [1].

Two main types of services are provided by the WSN which are query based and publish/subscribe based services. Each service has its own advantage as well as limitation. The pub/sub service is used in this application due to its advantage in user initiated services. This service is energy efficient which is very crucial factor in WSN. This has better scalability than other types of services. The user will receive the message automatically if he/she is subscribed to the service. Because of all these advantages, this service suits better compared to the query based service.

Even though it is possible to integrate many medical sensor to monitor, only one sensor is consider in this system for software implementation and Simulation purpose.

\section{E-Health Monitoring System}

EHMS is a system which gathers the information from the patient and process the data according to set of rules provided, then takes the necessary action if required. Fig 1 shows the architecture of the EHMS. The details of the EHMS is as explained

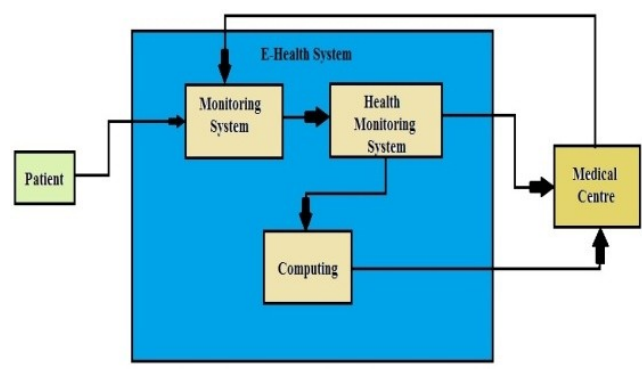

Fig 1 E-health monitoring system block diagram 


\subsection{Monitoring System}

Monitoring system collects the information from medical sensor from the patient. It then transfer this collected data to the Health monitoring system which is explained in the later part. Monitoring system compromises of the several health sensors to measures the Temperature, Blood pressure level, Heart Beat Rate and other characteristic of patient's. These sensors provide the collected information ultimately to the EHMS Application which will be explained in later part.

\subsection{Health Monitoring System}

Health monitoring system mainly focuses on one particular aspect like Heart Beat Rate or Blood pressure level, while the monitoring system collects the patient's health care data from the sensors. Health monitoring system uses set of standard format to read the data from monitoring system. It then manages to analyse the reading as per the information provided by medical schema. For Example a medical schema provides the information what action must be taken if the Heart Beat Rate indicates the abnormal reading. The Medical schema is provided by the concerned medical personal.

\subsection{Automatic Service}

Automatic services triggers the alert message to the medical personal, if there is any prediction of abnormal behaviour in patient character once the analysis in health monitoring system is completed.

\section{E-HMS Application}

EHMS application is done on the software which will be installed in the medical centre. EHMS application for this system is written in the .NET platform with the Visual Studio IDE. .NET is used due to main advantage which explained in later section. The Graphical User Interface (GUI) of this application is shown in Fig 2.

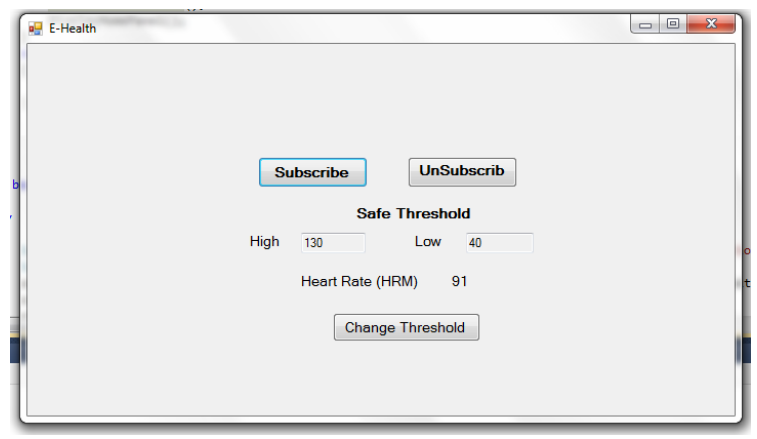

Fig 2 GUI of E-HMS Application

This EHMS application allows the doctor to subscribe or unsubscribe. A database is used to store the information of the doctor and also the threshold limit for processing. Validation are done in such a way that only the authorised medical personal are allowed to start the monitoring of the patient and also change the threshold limit if necessary. If the patient health data exceeds safe limit then alert message is delivered and display in the concerned authorised medical personal /Doctor.

\subsection{Use Case Diagram Of E-HMS Application}

Use case diagram is extensively used for Embedded System Design. Main program is divided into seven use cases for this application. Each use cases are programmed to perform set of task assigned to it. Use diagram for the EHMS application is shown in Fig 3. 


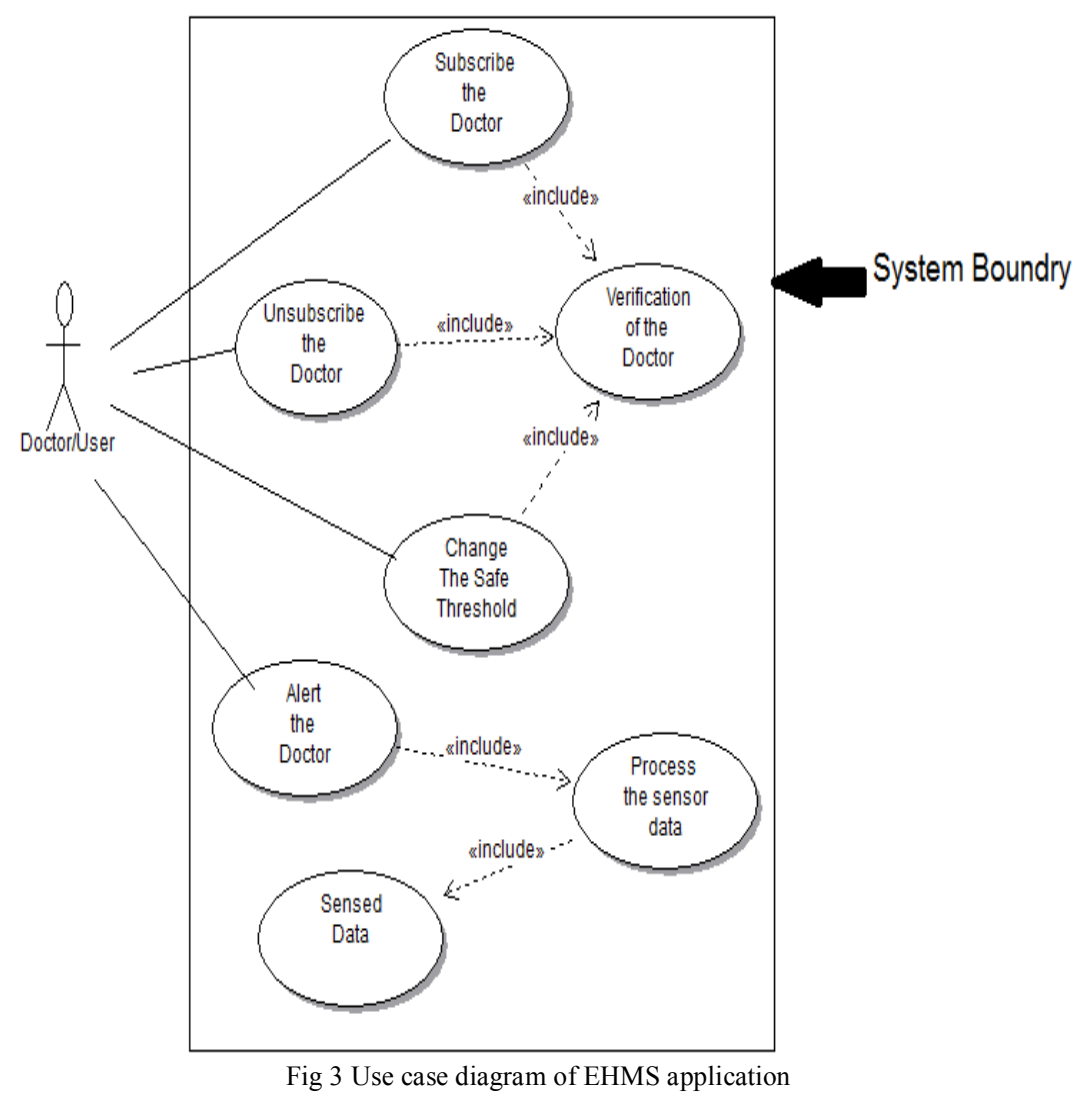

Seven use cases and their description are

ig 3 Use case diagram of EHMS application

Subscribe the doctor: Allow the medical personal to register his/her identity by storing the username and password for authentication.

Unsubscribe the doctor: An option to delete his/her identity from the database to minimize the memory usage. This option is advisable in case if health monitoring no longer needed.

Validation: This function is must step in EHMS Application. This ensures the authenticity of the medical personal to prevent any misuse of EHMS Application.

Change Threshold: This use case is written to change/alter the threshold limit of certain health characteristic is case advancement or collapse in patient health.

Process/Computing: This use function is to process the data from the monitoring system and also to process the request from medical application then it manages response for the EHMS application.

Alert Service: This use case function is to send an alert message to concerned medical personal for his attention in case of abnormal behaviour in patient i.e. if the monitored data is above or below the set limit provided by medical schema.

Sensed Data: This function collects the all data from the medical health sensor then feeds this data to health monitoring system.

\section{Simulation Approach}

Medical sensors are bit costly so it is advisable to build this system in simulation environment which practically replicates the original system to analyze the behaviour/result of the system \& EHMS Application. As mentioned previously EHMS Application is written in .NET platform in c\# language. Gumstix is used for the computational purposes.

Here simulation is carried out for generating the sensor data (some random number) which will be treated as sensor data. Gumstix work is to process, analyse and compute the data. This is also simulated.

Since the gumstix supports the .NET platform one could use the software written in this without changing. For better understanding of this system Activity diagram and its explanation will be given in later section. 


\subsection{Activity Diagram}

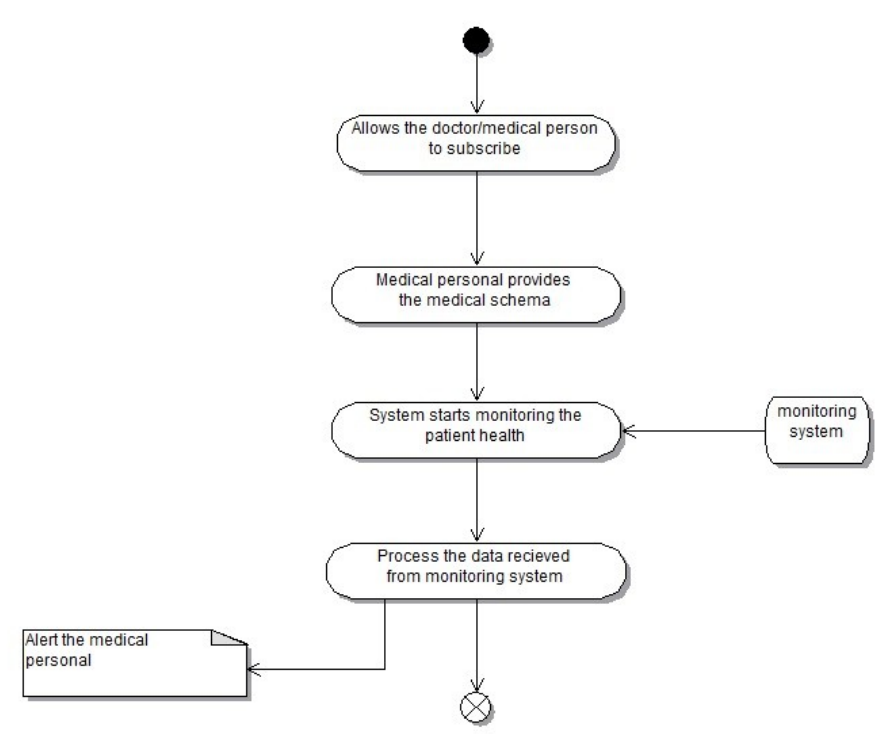

Fig 4 Activity Diagram

\subsection{Algorithm Of E-HMS}

- Initialisation of the monitoring process from authenticated medical personal from EHMS Application.

- EHMS continuously acquires data from sensors as per pre set time intervals.

- Computing device (Gumstix) process \& analyzes the data according to medical schema

- If the sensed data is off limit then it manages to alert the medical personal through message service in EHMS Application window.

\section{Simulation Result}

The result obtained is highly accurate and reliable. As previously mentioned simulation environment precisely replicates the actual system. The same accurate and precise results can be expected from the EHMS if it is implemented practically.

EHMS Application is fully secured, in other words this application prevents any accidents or unauthorised access, expect medical personal/ medical centre staff, who holds the authority to monitor the patient health. The alert services are sent via Internet protocol which will be received in medical centre/ EHMS application almost immediately.

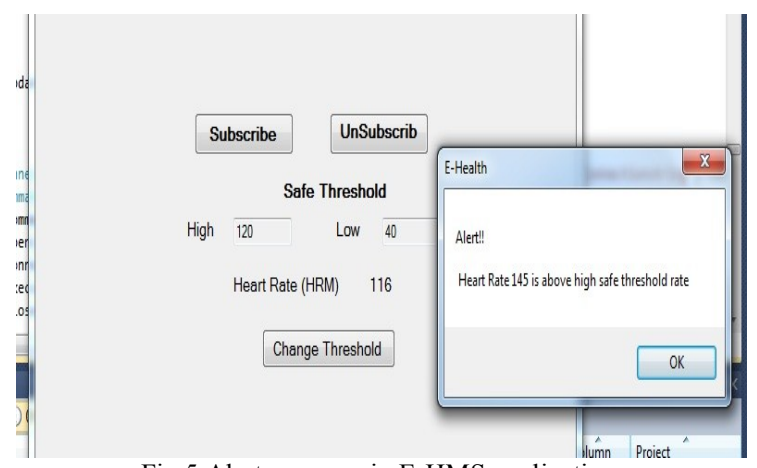

Fig 5 Alert message in E-HMS application

\section{Conclusion}

The simulation results suggest that it is possible to monitor the patient health characteristic remotely. EHMS application developed can be reliable and also it will be of very helpful for the medical personal. Since EHMS system along with this application minimizes the routine visits to the patients, by getting his/her attention towards the patients only if needed. Thus saving his/him time which in turn allows him/her to concentrate on more important things/works. This result also suggests that alert message delivery time is really fast, which is one of the major concerning factors in medical field. 
As the first step, this system is successfully simulated. Future work can be carried out by implementing this system in real time. This paper suggests some of the important hardware parts that are essential to the EHMS system. This application can be extended for more sensor, more patients.

\section{Reference}

[1] Terrance J. Dishongh, Michael McGrath (2010) Wireless sensor Network for Healthcare Application [Online] available from < http://site.ebrary.com/lib/coventry/docDetail.action?docID $=10359069>\left[5^{\text {th }}\right.$ May 2013].

[2] Tessa DANIEL, Elena GAURA (2008) Information Extraction from Large Scale WSN's: Approach and Research Issue [Online] available from $<$ http://wwwm. Coventry.ac.uk/researchnet/cogentcomputing/research/Documents/P 296.pdf $>$ [6 ${ }^{\text {th }}$ May 2013].

[3] Anon (n.d) Publish and Subscribe Pattern [Online] available from $<$ http://en.wikipedia.org/wiki/Publish/subscribe $>$ [6 ${ }^{\text {th }}$ May 2013].

[4] David Tam, Reza Azimi and Hans-Arno Jacobsen (n.d) Building content based System with Distributed Hash Tables [Online] available from $<$ http://www.eecg.toronto.edu/ tamda/dht/dht.pdf $>\left[6^{\text {th }}\right.$ May 2013].

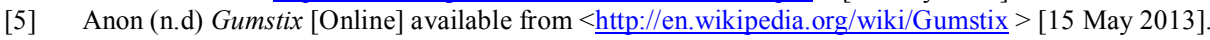

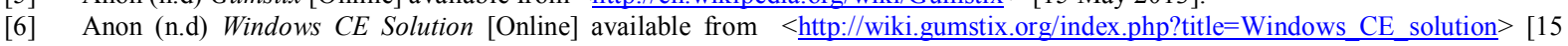
May 2013].

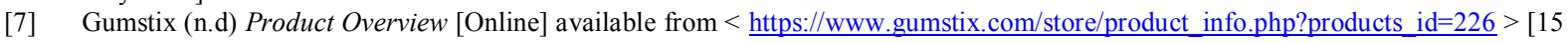
May 2013].

[8] OpenEmbedded (n.d) OpenEmbedded [Online] available from < http://www.openembedded.org/wiki/Main Page $>$ [15 May 2013]

[9] Gumstix (n.d) Software [Online] available from < https://www.gumstix.com/software.html $>$ [15 May 2013]. 